To the Editors:

\title{
Viral myositis caused by Epstein-Barr virus (EB virus) in children
}

We studied the aetiological agents responsible for causing a febrile illness associated with severe pain in calf muscles in children. All children presenting with a short febrile illness with difficulty in walking due to severe pain in the calf muscles were included in our study, done in the private and public sectors. The study was done between September 1995 and March 1996, and from April 1998 to December 1998. A majority of patients were ambulant. Leptospirosis was excluded on clinical grounds by absence of urinary symptoms, eye signs, hepatomegaly and myalgia at sites other than the calves. Virological studies were performed at the MRI. EBV IgM was estimated in the acute or convalescent samples using an Elisa test (Behring) and EBV specific IgM. The cutoff point used was 0.02 . Other viruses tested for included dengue $D_{2}$ and $D_{3}$ (haemagglutination inhibition test), influenza $A$ and $B$ (haemagglutination inhibition test), Hanta (indirect fluorescent test) and enteroviruses in stool samples using HEp2 and RD cells.

A total of 18 children (11 boys) were studied. 4 children were under 5 years. 12 were between 5 and 10 years and 2 were over 10 years. All children had fever, difficulty in walking and pain and tenderness over the calf muscles. They were treated with paracetamol with or without oral diazepam, and all recovered within a week. Some parents were apprehensive because of similarities with poliomyelitis, but were reassured when they recovered.

All patients had elevated serum CPK levels. The highest level recorded was 19800 units/ml (normal range 0 to 199 units/ml). The CPK levels returned to normal within 2 weeks.
Virological studies revealed a significantly positive EBV IgM titre in the acute sample in 10 patients. It was indeterminate in the acute sample in 5, and of these the convalescent sample was positive in 2 patients. The tests for influenza, Hanta and enterovirus were nagative in all patients. One patient had a dengue $D_{2}$ and $D_{3}$ titre of $1 / 80$ in both acute and convalescent samples, but the convalescent samples was positive for EBV IgM as well.

Viral myositis is a well documented entity which is not uncommon in Sri Lankan children. Some parents worry because of similarities with poliomyelitis. It is a self-limiting illness which requires only symptomatic treatment and reassurance. Estimation of serum CPK levels is useful in establishing the diagnosis. EB virus seems to play a significant role in its aetiology.

In previous studies of benign acute childhood myositis, infection with influenza A and B virus has been incriminated (1). In that study myositis was associated with the initial infection rather than a subsequent infection. In another study, no virus could be identified. (2). Although diverse clinical manifestations are attributed to infection with EBV, a benign acute myositis has not been documented in children. Significantly, in this study $60 \%$ of the children had evidence of initial infection with EBV.

We thank Ms. Hemamali Pathmathilake for typing the manuscript.

\section{References}

1. Ruff RI, Secrist D. Viral studies in benign acute childhood myositis. Achives of Neurology 1982; 39: 261-3.

2. Antony JH, Procopis PG, Ouvrier RA. Benign acute childhood myositis. Neurology 1979; 29: 1968-7.

S P Lamabadusuriya, Professor and Head, Department of Paediatrics, Faculty of Medicine, University of Colombo; Nalini Witharana, Virologist, and L D Preethimala, Medical Laboratory Technologist, Medical Research Institute, Colombo. (Correspondence SPL, telephone +941695300) 